\title{
Measuring Density Functional Parameters from Electron Diffraction Patterns
}

\author{
Ding Peng ${ }^{*}$ and Philip N.H. Nakashima $\odot^{\dagger}$ \\ Department of Materials Science and Engineering, Monash University, Victoria 3800, Australia
}

(Received 17 October 2020; revised 13 January 2021; accepted 15 March 2021; published 29 April 2021)

\begin{abstract}
We integrate density functional theory (DFT) into quantitative convergent-beam electron diffraction (QCBED) to create a synergy between experiment and theory called QCBED-DFT. This synergy resides entirely in the electron density which, in real materials, gives rise to the experimental CBED patterns used by QCBED-DFT to refine DFT model parameters. We use it to measure the Hubbard energy $U$ for two strongly correlated electron systems, $\mathrm{NiO}$ and $\mathrm{CeB}_{6}(U=7.4 \pm 0.6 \mathrm{eV}$ for $d$ orbitals in $\mathrm{NiO}$ and $U=$ $3.0 \pm 0.6 \mathrm{eV}$ for $f$ orbitals in $\left.\mathrm{CeB}_{6}\right)$, and the boron position parameter $x$ for $\mathrm{CeB}_{6}(x=0.1992 \pm 0.0003)$. In verifying our measurements, we demonstrate an accuracy test for any modeled electron density.
\end{abstract}

DOI: 10.1103/PhysRevLett.126.176402

Density functional theory (DFT) is ubiquitous in materials science because it models electron densities and all resultant materials properties with useful accuracy at reasonable computational cost. The theory is exact-the exact functional of the exact density will give the exact energy of the system [1-4]; however, the exact functional is undefined. Approximating it with functionals parametrized to reproduce energies of well-characterized systems has raised concerns that DFT "is straying from the path toward the exact functional" $[3,4]$ because reproducing the correct energies does not imply reproduction of the true electron density [1-4].

Quantitative convergent-beam electron diffraction (QCBED) is an experimental pattern-matching technique, unsurpassed in accuracy and precision when measuring electron densities [5-53]. We have integrated DFT into QCBED to fit experimental CBED patterns from the actual electron density in real materials, with simulated CBED patterns calculated from DFT-modeled electron densities. Our method, QCBED-DFT, refines DFT model parameters without comparing energies or properties at all, confining the refinements to electron densities alone.

The electron density $\rho(\mathbf{r})$ is the dominant determinant of materials properties and, if accurately known by other means, can provide a three-dimensional constraint for DFT, as emphasized by Kohn [1]. In this spirit, frozen-density embedding theory uses x-ray diffraction to constrain the Hohenberg-Kohn functional [54]. Analogs in many-body wave function methods have given rise to the field of

Published by the American Physical Society under the terms of the Creative Commons Attribution 4.0 International license. Further distribution of this work must maintain attribution to the author(s) and the published article's title, journal citation, and DOI. x-ray-constrained wave functions [53,55-60]. In both cases, the accuracy of the x-ray diffraction data is critical.

Extinction [61] in x-ray diffraction can be a problematic error resulting from multiple scattering in the context of a kinematic scattering analysis. In contrast, QCBED employs a full dynamical scattering treatment, owing to the very strong dynamical scattering of electrons by matter. The latter gives rise to the very detailed intensity distributions in CBED patterns (see Figs. 1 and 2 and Supplemental Material, Tables S4 and S8 [62]) that QCBED typically matches at the rate of $\sim 10^{3}$ independent intensities per reflection. This leads to parameter oversampling by approximately 3 orders of magnitude. Furthermore, the routine subnanometer spatial selectivity in transmission electron microscopy means that very small volumes of perfect crystal ( $\sim 10^{9}$ times smaller than in x-ray diffraction) can be selected for CBED pattern acquisition. These factors contribute to the very high precision and accuracy in electron density measurements for which QCBED is renowned [5-53].

In QCBED, an experimental point spread function (PSF)-corrected [82] CBED pattern is fitted with a theoretically calculated one by refining the parameters to which the diffracted intensities are most sensitive. Recent methods incorporate angular differentiation to remove most of the inelastic signal that impedes matching with an elastic electron scattering theory [42-44,51,53]. In Figs. 1 and 2, where the differences between conventional QCBED (CQCBED) and QCBED-DFT are illustrated, the experimental data are labeled $I_{\text {expt }}^{\prime}$ and the refined calculated intensities are marked $I_{\text {calc }}^{\prime}$.

The crystal potential $V(\mathbf{r})$ is essential to the calculation of CBED patterns and its Fourier coefficients (structure factors) $V_{h k l}$ are exactly interconvertible with those of $\rho(\mathbf{r})$, i.e., $F_{h k l}$, by the Mott-Bethe formula $[83,84]$. In CQCBED, an independent atom model (IAM), which ignores interatomic bonding, is used and the refined parameters include the bonding-affected $V_{h k l}$. 

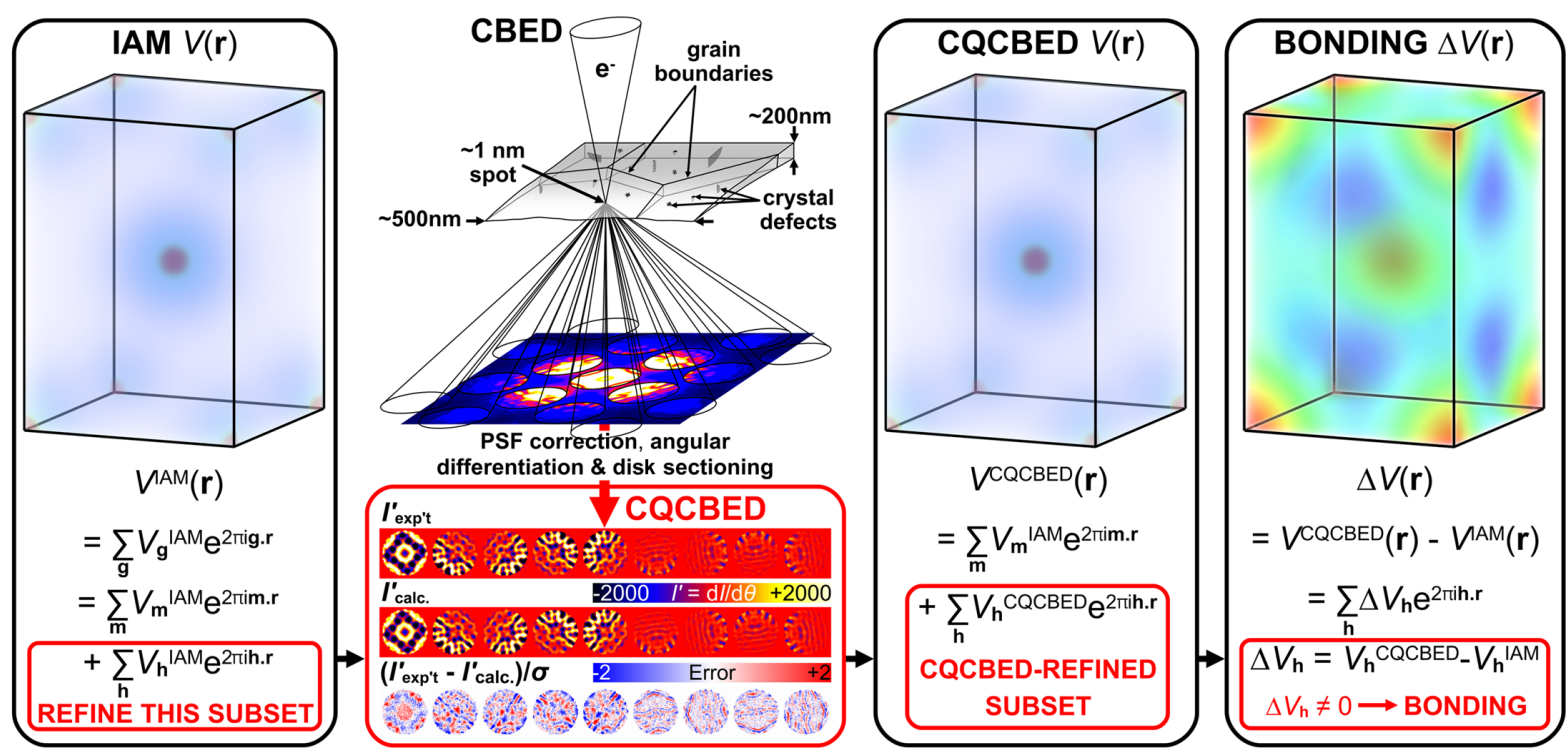

FIG. 1. Conventional QCBED (CQCBED). A few parameters are adjusted to fit a calculated CBED pattern to an experimental one, including the structure factors $V_{h}$, to which the intensities are most sensitive. These form a very small subset of all structure factors $V_{g}$, required for a full dynamical electron scattering calculation. The unrefined remainder $V_{\mathbf{m}}$ are obtained from an IAM, i.e., $V_{\mathbf{m}}^{\mathrm{IAM}}$. As the refinement progresses, the modified structure factors that start with IAM values $V_{h}^{\mathrm{IAM}}$ take on new values $V_{h}^{\mathrm{CQCBED}}$. The bonding potential is the difference between the CQCBED-refined and IAM potentials and is equivalent to computing the Fourier sum using the difference structure factors $\Delta V_{h}$. Here, the bonding potential in aluminum from [43] has been used as an example of CQCBED.

The differences between the CQCBED-measured structure factors and their IAM counterparts constitute a set of difference structure factors that quantify the bonding potential $\Delta V(\mathbf{r})$ (Fig. 1). This is accurate only if (i) all bonding-affected structure factors have been refined, and (ii) the IAM represents isolated atoms accurately.
By replacing the IAM with DFT, QCBED-DFT treats the material as an ensemble of bonded atoms, not independent ones. Instead of refining small subsets of structure factors, QCBED-DFT refines DFT model parameters, altering the simulated electron density in real space and therefore changing all structure factors used to calculate CBED

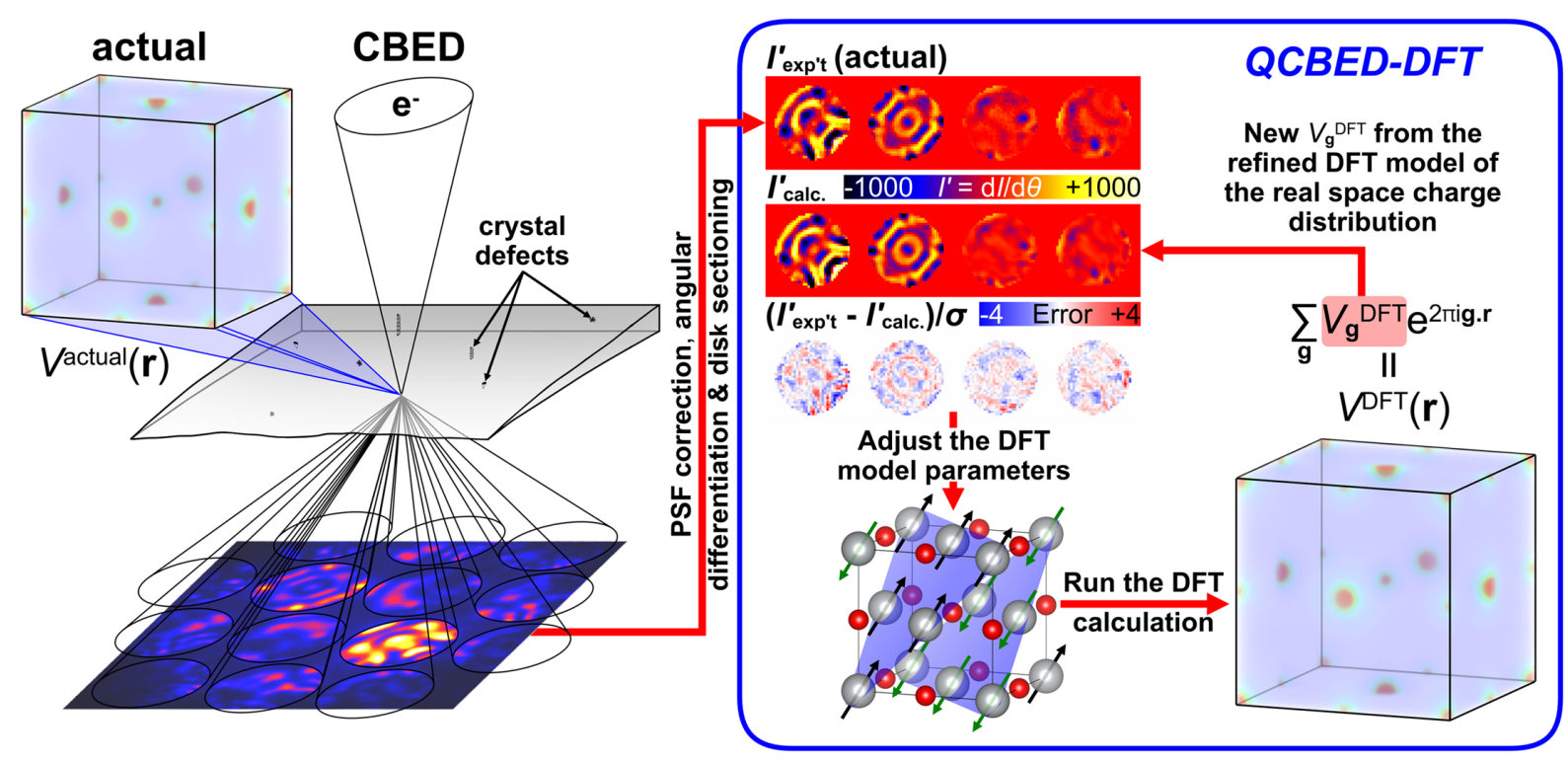

FIG. 2. QCBED-DFT. The DFT model parameters are refined which, when adjusted, change the simulated electron density in real space, thereby changing all structure factors $V_{g}^{\mathrm{DFT}}$ of the crystal potential returned to the electron scattering calculations. By optimizing the fit between the calculated and experimental CBED patterns, the DFT model parameters are refined (see Supplemental Material [62] for details). The present example involves antiferromagnetic NiO-a subject of this Letter. 
(a)

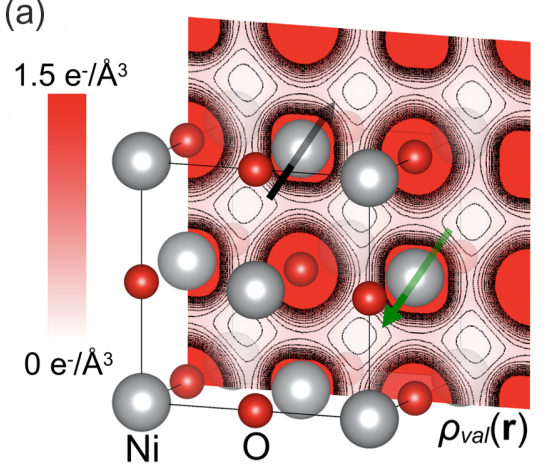

(b)

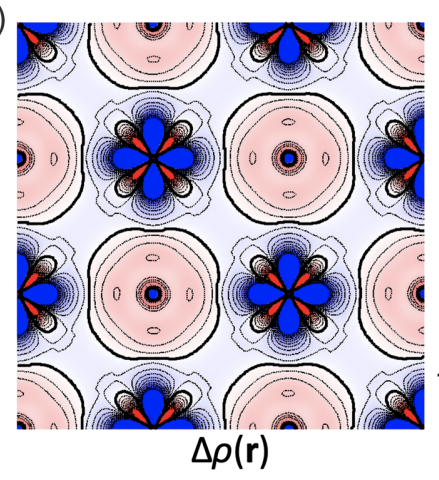

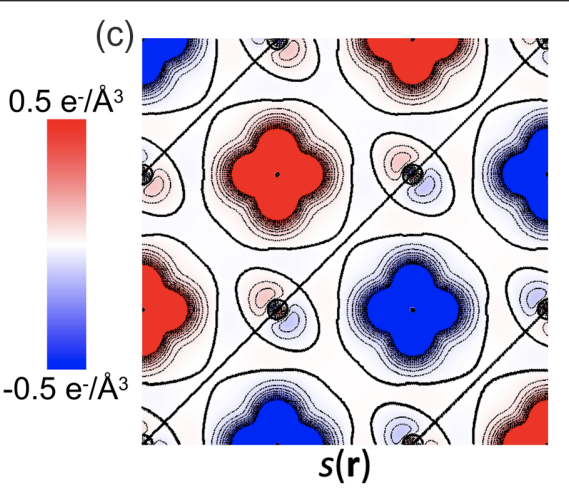

FIG. 3. The electronic structure of $\mathrm{NiO}$ determined by QCBED-DFT with $U=7.4 \mathrm{eV}$ and $J=0.95 \mathrm{eV}$ for $d$ orbitals $[24,91,98]$ at $T=0 \mathrm{~K}$. The valence electron density $\rho_{\text {val }}(\mathbf{r})$ is mapped in the (020) plane with a contour interval of $0.1 e^{-} \AA^{-3}$ (a). Nearest-neighbor nickel atoms (gray) have opposite spins indicated by the black and green arrows parallel and antiparallel to [112]. The bonding and electron spin densities, (b) $\Delta \rho(\mathbf{r})$ and (c) $s(\mathbf{r})$, respectively, are plotted for the same region as (a) $\rho_{\text {val }}(\mathbf{r})$, but with a contour interval of $0.05 e^{-} \AA^{-3}$ and the zero contour thickened.

patterns (Fig. 2). At present, QCBED-DFT uses Bloch wave code $[9,85]$ for the CBED intensity calculations and calls WIEN2K [86] for the DFT-calculated crystal potential as input (see Supplemental Material [62] for details).

Instead of testing density functionals by comparing system energies and materials properties, QCBED-DFT interrogates $\rho(\mathbf{r})$ directly because the experimental CBED patterns being matched are a direct consequence of $V(\mathbf{r})$, and thus $\rho(\mathbf{r})$, in the actual material.

While QCBED experimentally constrains DFT parameters, the $V(\mathbf{r})$ calculated by DFT at each iteration of QCBED-DFT represents the material better than an IAM [87], increasing the accuracy of QCBED. Furthermore, DFT provides the theoretical framework for translating the QCBED-DFT-optimized electron density into a large suite of materials properties, assuming that the functional and calculation protocols are sufficiently accurate for the material being modeled $[2,53,86,88-90]$.

Strongly correlated electron systems have challenged DFT $[2,88,91]$, and one way of dealing with electron correlations has been to add the Hubbard energy parameter $U$ into the exchange-correlation functional $[2,88,91]$. In our investigations of $\mathrm{NiO}$ and $\mathrm{CeB}_{6}$, we used the PerdewBurke-Ernzerhof (PBE) generalized gradient approximation (GGA) $[86,92]$ in a GGA(PBE) $+U$ configuration and we refined $U$. This was done within the augmented plane wave plus local orbitals (APW + lo) regime of WIEN2k [86]. For $\mathrm{CeB}_{6}$, we also refined the positions of the boron atoms because of significant discrepancies in the literature [93-97]. All experimental details, DFT and QCBED settings within QCBED-DFT, refinement outputs, materials properties, and electronic structure morphologies are discussed in the Supplemental Material [62].

Figure 2 shows a typical example of a QCBED-DFT refinement from the nine that were performed for $\mathrm{NiO}$ (see Tables S1-S4 in [62]). All of them involved the refinement of only eight parameters, including the Hubbard energy $U$, to fit 3026 independent intensities per pattern. The nine CBED patterns came from regions of different specimen thickness ranging from 1288 to $2001 \AA$ (see Tables S1-S3 in [62]) and were collected with $202.7 \pm 0.2 \mathrm{keV}$ electrons incident near $\langle 011\rangle$. We report a value of $U=7.4 \pm 0.6 \mathrm{eV}$ for $d$ orbitals in NiO from our QCBED-DFT refinements, with a Hund exchange parameter of $J=0.95 \mathrm{eV}$ $[24,91,98]$. This result is within the range of $U=$ 4.6-8.0 eV reported previously [91,98-105].

Figure 3 presents the QCBED-DFT-optimized electronic structure of $\mathrm{NiO}$ at $T=0 \mathrm{~K}$. Figures 3(a)-3(c) show that the valence and bonding electron densities, $\rho_{\mathrm{val}}(\mathbf{r})$ [Fig. 3(a)] and $\Delta \rho(\mathbf{r})$ [Fig. 3(b)], respectively, are insensitive to the opposed magnetic moments of nearest-neighbor nickel atoms, while the electron spin density $s(\mathbf{r})$ [Fig. 3(c)] distinguishes them very clearly, as expected.

While CQCBED can determine $\Delta \rho(\mathbf{r})$ accurately (given an accurate IAM), the determination of $\rho_{\text {val }}(\mathbf{r})$ would require an accurate independent ion model. WIEN2k uses the muffin-tin model, providing separate access to $\rho_{\text {val }}(\mathbf{r})$ by virtue of this formalism [86]. On its own, CQCBED cannot determine $s(\mathbf{r})$ because CBED is insensitive to electron spin. Revealing $\rho_{\text {val }}(\mathbf{r})$ and $s(\mathbf{r})$ is a benefit of the theoretical framework provided by DFT in the context of the electron density born out of that same formalism. However, in contrast to stand-alone DFT, the electron density in the present case has been refined against experimental CBED patterns, which are a direct consequence of the actual $\rho(\mathbf{r})$ in the real material.

For $\mathrm{CeB}_{6}$, in addition to refining $U$, we also refined the boron position parameter $x$ [Figs. 4(a) and 4(b) and Supplemental Material, Fig. S5a [62]] because of significant discrepancies in previously reported values, i.e., $x=0.200 \pm 0.002$ [93-97] (see Table S5 in [62]).

We analyzed 14 CBED patterns with QCBED-DFT, seven collected with $121.3 \pm 0.2 \mathrm{keV}$ electrons incident near $\langle 001\rangle$ and seven collected with $202.7 \pm 0.2 \mathrm{keV}$ 

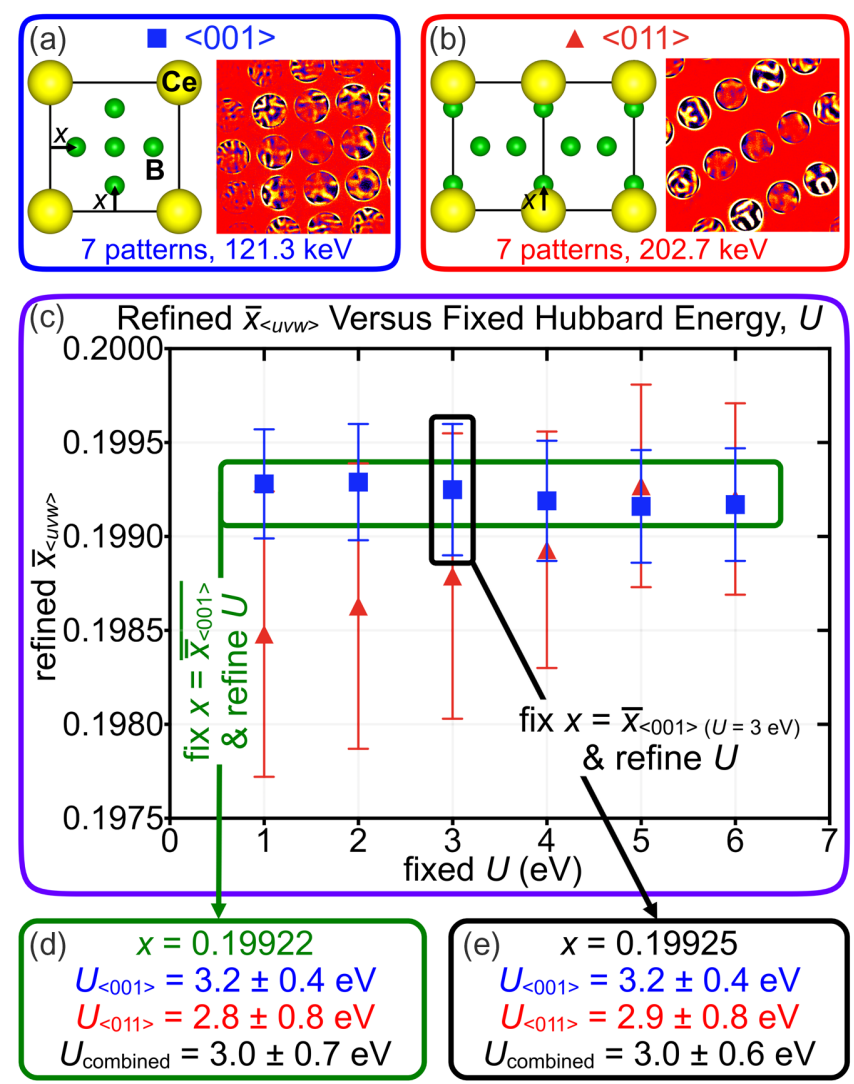

FIG. 4. Determination of the atomic structure parameter $x$ and the Hubbard energy $U$ for $f$ orbitals in $\mathrm{CeB}_{6}$ using QCBED-DFT. The boron atom positions are defined by $x$ (a),(b). Sets of seven CBED patterns from (a) near $\langle 001\rangle$ and (b) $\langle 011\rangle$ were matched by fixing $U$ and refining $x$. The mean values of $x$ from each zone axis, $\bar{x}_{\langle u v w\rangle}$, are graphed with their uncertainties (c) for each value of $U$ (see Tables S6 and S7 in [62]). Fixing $x=\bar{x}_{\langle 001\rangle}=0.19922$ (the mean of $\bar{x}_{\langle 001\rangle}$ over all values of $U$ ), $U$ was then refined and results for the $\langle 001\rangle \mathrm{CBED}$ data $\left(U_{\langle 001\rangle}\right)$, the $\langle 011\rangle$ data $\left(U_{\langle 011\rangle}\right)$, and all data combined $\left(U_{\text {combined }}\right)$ are reported $(\mathrm{d})$. This was repeated for $x=\bar{x}_{\langle 001\rangle(U=3 \mathrm{eV})}=0.19925$ (e).

electrons incident near $\langle 011\rangle$. All patterns came from regions of different specimen thickness ranging from 1186 to $1719 \AA$ (see Tables S6 and S7 in the Supplemental Material [62]). This experimental variety tests the precision of the results more robustly than repeated measurements under the same conditions.

Our refinements of $x$ and $U$ involved two stages with only one parameter refined during each stage in order to assess and mitigate parameter correlations.

In stage one, all $14 \mathrm{CBED}$ patterns were matched by refining only $x$, while $U$ was fixed at values from 1 to $6 \mathrm{eV}$ in increments of $1 \mathrm{eV}$. The mean $x$ obtained from the seven refinements near each zone axis, $\bar{x}_{\langle u v w\rangle}$, is plotted for each fixed value of $U$, for each of $\langle 001\rangle$ and $\langle 011\rangle$, in Fig. 4(c). The independence of $x$ from $U$ is evident in all refinements using CBED patterns near $\langle 001\rangle$, while they appear to be linearly correlated in the refinements using near $\langle 011\rangle$ data.
This is probably due to the manifestation of $x$ in two noncollinear directions perpendicular to $\langle 001\rangle$ [Fig. 4(a)] as opposed to just one such appearance perpendicular to $\langle 011\rangle$ [Fig. 4(b)]. Furthermore, all boron atom columns in $\langle 001\rangle$ projections are well separated from cerium columns, while this is not the case for $\langle 011\rangle$. As a first estimate of $x$, we used only the results from the $\langle 001\rangle$ data, averaging over $\bar{x}_{\langle 001\rangle}$ for all fixed values of $U$ to get $\bar{x}_{\langle 001\rangle}=0.19922$.

Stage two involved fixing $x=0.19922$ and refining $U$ using all 14 CBED patterns. The results are summarized in Fig. 4(d) and suggest that $U=3 \mathrm{eV}$. We then repeated the refinements of $U$ with $x$ fixed at $\bar{x}_{\langle 001\rangle(U=3 \mathrm{eV})}$, i.e., $x=0.19925$. The results of this final set of refinements are summarized in Fig. 4(e) and are not significantly different to the preceding results in Fig. 4(d).

We conclude that $x=\bar{x}_{\langle 001\rangle(U=3 \mathrm{eV})}=0.1992 \pm 0.0003$ and $U=3.0 \pm 0.6 \mathrm{eV}$ for $f$ orbitals in $\mathrm{CeB}_{6}$. This is in agreement with Sato $(x=0.19923 \pm 0.00006)$ [93], Blomberg et al. $\quad(x=0.1992 \pm 0.0001)$ [95], and Streltsov et al. $(x=0.1995 \pm 0.0003)$ [96] for $x$, while our refined value of $U$ agrees with Barman et al. [106]. The resulting $\Delta \rho(\mathbf{r})$ is examined in detail in [62] (Fig. S5 and surrounding discussion [62]).

Quantum crystallography [53] is always in pursuit of more accurate electron densities, raising the question of how can one test the accuracy of a modeled $\rho(\mathbf{r})$ ? The answer is in the form of CQCBED (Fig. 1).

In CQCBED, discrepancies between the modeled $\rho(\mathbf{r})$ and the actual $\rho(\mathbf{r})$, from which the experimental CBED patterns arise, will cause individual structure factors to change from the modeled values in order to minimize the pattern mismatch. This is how CQCBED with an IAM has been applied to date- to measure changes in structure factors from IAM values due to bonding (Fig. 1) [5-53]. If the IAM in CQCBED was replaced with the true $\rho(\mathbf{r})$, then the refinement of individual structure factors would result in no changes from their modeled values. We used this principle to test the accuracy of the QCBED-DFTdetermined $\rho(\mathbf{r})$.

We performed two sets of CQCBED refinements for $\mathrm{NiO}$ and $\mathrm{CeB}_{6}$. In the first set, we used the $V(\mathbf{r})$ [and thus $\rho(\mathbf{r})$ ] resulting from our QCBED-DFT refinements, and we label this CQCBED-DFT for the purposes of the present discussion. For comparison, we also performed a second set of CQCBED refinements using the standard IAM [107-109] for $V(\mathbf{r})$ and thus $\rho(\mathbf{r})$. We label this set of refinements CQCBED-IAM. The individually refined structure factors in CQCBED-DFT and CQCBED-IAM were not only those to which the experimental CBED patterns were most sensitive, but were also limited to those with low parameter correlations.

Figure 5 (see also Table S11 in [62]) plots the CQCBEDDFT- and CQCBED-IAM-refined electron density structure factors $F_{h k l}$ converted from potential structure factors $V_{h k l}$, using the Mott-Bethe formula $[83,84]$ and normalized 


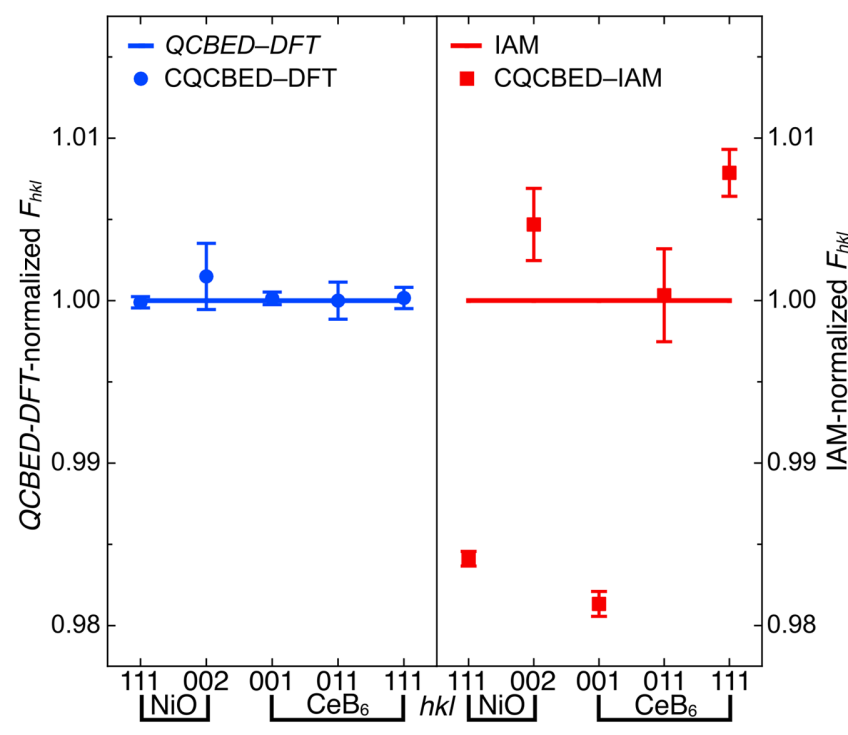

FIG. 5. A comparison of electron density structure factors $F_{h k l}$ refined by CQCBED with different reference electronic structure models for $\mathrm{NiO}$ and $\mathrm{CeB}_{6}$. All points represent the refined $F_{h k l}$ normalized with respect to the corresponding model values. The horizontal lines represent each model where QCBED-DFT corresponds to DFT [APW + lo: $\mathrm{GGA}(\mathrm{PBE})+U,(U=7.4$ and $J=0.95 \mathrm{eV}$ for $\mathrm{NiO}),(U=3 \mathrm{eV}, J=0 \mathrm{eV}$, and $x=$ 0.19925 for $\mathrm{CeB}_{6}$ )], as determined by our QCBED-DFT refinements, and the IAM is the current International Union of Crystallography standard [107-109]. All $F_{h k l}$ correspond to the temperatures of the experiments and were converted from $V_{h k l}$ by the Mott-Bethe formula [83,84]. See Table S11 in [62] for the actual values of $V_{h k l}$ and $F_{h k l}$.

by their modeled counterparts at the temperatures of the CBED experiments, $T=110$ and $T=298 \mathrm{~K}$ for $\mathrm{NiO}$ and $\mathrm{CeB}_{6}$, respectively. The horizontal lines at 1 on the ordinate axes represent the QCBED-DFT-determined $\rho(\mathbf{r})$ (blue) and IAM $\rho(\mathbf{r})$ (red). The large deviations of the IAMnormalized, CQCBED-IAM-refined $F_{h k l}$ from unity indicate the relative magnitudes of bonding effects on each of the structure factors. In contrast, the QCBED-DFT-normalized, CQCBED-DFT-refined $F_{h k l}$ for both $\mathrm{NiO}$ and $\mathrm{CeB}_{6}$ have not departed from the QCBED-DFT-modeled $\rho(\mathbf{r})$. This is evidence that, at least for $\mathrm{NiO}$ and $\mathrm{CeB}_{6}$, $\mathrm{GGA}(\mathrm{PBE})+U[U=7.4 \pm 0.6 \mathrm{eV}$ and $J=0.95 \mathrm{eV}$ for $d$ orbitals in NiO; $U=3.0 \pm 0.6 \mathrm{eV}$ and $J=0 \mathrm{eV}$ for $f$ orbitals in $\mathrm{CeB}_{6}$ ] generates electron densities that are as close to reality in these materials as may be determined from experimental CBED patterns.

The uncertainties in the CQCBED-DFT-refined structure factors are consistently smaller than those of CQCBED-IAM, with improvements in precision as much as threefold in some cases (see Table S11 in the Supplemental Material [62]). Replacing the IAM with a more accurate DFT model is bound to improve the accuracy of the electron scattering calculations within QCBED, yielding more consistent matches of calculated and experimental CBED patterns and, therefore, reduced parameter uncertainties.

Assessing the accuracy of modeled electron densities and refining DFT model parameters using QCBED-DFT, demonstrated here, has a broader scope than the present focus on $\mathrm{GGA}(\mathrm{PBE})+U$. Future work should aim to test other functionals and contribute to the development of new ones. Matching 3D electron densities is more constrained than matching energies alone, making QCBED-DFT a potentially useful method in the search for the exact density functional.

Professor A. W. S. Johnson was particularly influential in this research and helped procure the $\mathrm{CeB}_{6}$ single crystal, together with Mr. P. Hanan. Dr. H. Cheng helped in preparing the $\mathrm{NiO}$ specimen. Professor J.-M. Zuo shared his REFINECB5 code, which forms a significant component of the QCBED-DFT package. Dr. A. E. Smith guided and trained D. P. in the use of WIEN2k. Professor J. Etheridge FAA reviewed our manuscript and gave us valuable advice. Associate Professor L. Bourgeois and Associate Professor M. Weyland provided microscopy training, expertise, and advice. Dr. T. Liu rendered practical help with computing. We thank all of the above and acknowledge the assistance at the Monash Centre for Electron Microscopy, Monash University, a node of Microscopy Australia, established under the Commonwealth Government's National Collaborative Research Infrastructure Strategy (NCRIS). P. N. thanks the Australian Research Council for funding (FT110100427 and DP210100308).

*Ding.Peng@ntnu.no

Also at Department of Physics, Norwegian University of Science and Technology (NTNU), Trondheim, Norway.

Philip.Nakashima@monash.edu

[1] W. Kohn, Rev. Mod. Phys. 71, 1253 (1999).

[2] R. O. Jones, Rev. Mod. Phys. 87, 897 (2015).

[3] M. G. Medvedev, I. S. Bushmarinov, J. Sun, J. P. Perdew, and K. A. Lyssenko, Science 355, 49 (2017).

[4] M. Korth, Angew. Chem., Int. Ed. Engl. 56, 5396 (2017).

[5] J. M. Zuo, J. C. H. Spence, and M. O'Keeffe, Phys. Rev. Lett. 61, 353 (1988).

[6] D. M. Bird and M. Saunders, Ultramicroscopy 45, 241 (1992).

[7] J. C. H. Spence, Acta Crystallogr. Sect. A 49, 231 (1993).

[8] J. M. Zuo, J. C. H. Spence, J. Downs, and J. Mayer, Acta Crystallogr. Sect. A 49, 422 (1993).

[9] J. M. Zuo, Acta Crystallogr. Sect. A 49, 429 (1993).

[10] C. Deininger, G. Necker, and J. Mayer, Ultramicroscopy 54, 15 (1994).

[11] M. Saunders, D. M. Bird, N. J. Zaluzec, W. G. Burgess, A. R. Preston, and C. J. Humphreys, Ultramicroscopy 60, 311 (1995).

[12] K. Tsuda and M. Tanaka, Acta Crystallogr. Sect. A 51, 7 (1995).

[13] R. Holmestad, J. M. Zuo, J. C. H. Spence, R. Høier, and Z. Horita, Philos. Mag. A 72, 579 (1995).

[14] J. M. Zuo and A. L. Weickenmeier, Ultramicroscopy 57, 375 (1995). 
[15] L. M. Peng and J. M. Zuo, Ultramicroscopy 57, 1 (1995).

[16] M. Saunders, D. M. Bird, O. F. Holbrook, P. A. Midgley, and R. Vincent, Ultramicroscopy 65, 45 (1996).

[17] P. A. Midgley and M. Saunders, Contemp. Phys. 37, 441 (1996).

[18] C. Birkeland, R. Holmestad, K. Marthinsen, and R. Høier, Ultramicroscopy 66, 89 (1996).

[19] J. M. Zuo, M. O'Keeffe, P. Rez, and J. C. H. Spence, Phys. Rev. Lett. 78, 4777 (1997).

[20] J. M. Zuo, M. Kim, M. O'Keeffe, and J. C. H. Spence, Nature (London) 401, 49 (1999).

[21] M. Saunders, A. G. Fox, and P. A. Midgley, Acta Crystallogr. Sect. A 55, 471 (1999).

[22] M. Saunders, A. G. Fox, and P. A. Midgley, Acta Crystallogr. Sect. A 55, 480 (1999).

[23] K. Tsuda and M. Tanaka, Acta Crystallogr. Sect. A 55, 939 (1999).

[24] S. L. Dudarev, L. M. Peng, S. Y. Savrasov, and J. M. Zuo, Phys. Rev. B 61, 2506 (2000).

[25] V. A. Streltsov, P. N. H. Nakashima, and A. W. S. Johnson, J. Phys. Chem. Solids 62, 2109 (2001).

[26] K. Tsuda, Y. Ogata, K. Takagi, T. Hashimoto, and M. Tanaka, Acta Crystallogr. Sect. A 58, 514 (2002).

[27] B. Jiang, J. M. Zuo, N. Jiang, M. O'Keeffe, and J. C. H. Spence, Acta Crystallogr. Sect. A 59, 341 (2003).

[28] J. Friis, B. Jiang, J. C. H. Spence, and R. Holmestad, Microsc. Microanal. 9, 379 (2003).

[29] V. A. Streltsov, P. N. H. Nakashima, and A. W. S. Johnson, Microsc. Microanal. 9, 419 (2003).

[30] B. Jiang, J. M. Zuo, J. Friis, and J. C. H. Spence, Microsc. Microanal. 9, 457 (2003).

[31] J. Friis, G. K. H. Madsen, F. K. Larsen, B. Jiang, K. Marthinsen, and R. Holmestad, J. Chem. Phys. 119, 11359 (2003).

[32] Y. Ogata, K. Tsuda, Y. Akishige, and M. Tanaka, Acta Crystallogr. Sect. A 60, 525 (2004).

[33] B. Jiang, J. Friis, R. Holmestad, J. M. Zuo, M. O’Keeffe, and J. C. H. Spence, Phys. Rev. B 69, 245110 (2004).

[34] J. Friis, B. Jiang, J. C. H. Spence, K. Marthinsen, and R. Holmestad, Acta Crystallogr. Sect. A 60, 402 (2004).

[35] J. M. Zuo, Rep. Prog. Phys. 67, 2053 (2004).

[36] P. N. H. Nakashima, J. Appl. Crystallogr. 38, 374 (2005).

[37] J. Friis, B. Jiang, K. Marthinsen, and R. Holmestad, Acta Crystallogr. Sect. A 61, 223 (2005).

[38] P. N. H. Nakashima, Phys. Rev. Lett. 99, 125506 (2007).

[39] K. Tsuda, D. Morikawa, Y. Watanabe, S. Ohtani, and T. Arima, Phys. Rev. B 81, 180102(R) (2010).

[40] X. H. Sang, A. Kulovits, and J. M. K. Wiezorek, Acta Crystallogr. Sect. A 66, 685 (2010).

[41] X. H. Sang, A. Kulovits, and J. M. K. Wiezorek, Acta Crystallogr. Sect. A 66, 694 (2010).

[42] P. N. H. Nakashima and B. C. Muddle, Phys. Rev. B 81, 115135 (2010).

[43] P. N. H. Nakashima, A. E. Smith, J. Etheridge, and B. C. Muddle, Science 331, 1583 (2011).

[44] P. A. Midgley, Science 331, 1528 (2011).

[45] X. H. Sang, A. Kulovits, and J. M. K. Wiezorek, Acta Crystallogr. Sect. A 67, 229 (2011).

[46] X.H. Sang, A. Kulovits, G. F. Wang, and J. M. K. Wiezorek, Philos. Mag. 92, 4408 (2012).
[47] P. N. H. Nakashima, Opt. Lett. 37, 1023 (2012).

[48] X. H. Sang, A. Kulovits, G. F. Wang, and J. M. K. Wiezorek, J. Chem. Phys. 138, 084504 (2013).

[49] X. H. Sang, A. Kulovits, and J. M. K. Wiezorek, Ultramicroscopy 126, 48 (2013).

[50] D. Peng and P. N. H. Nakashima, J. Appl. Crystallogr. 50, 602 (2017).

[51] P. N. H. Nakashima, Structural Chemistry 28, 1319 (2017).

[52] J. M. Zuo and J. C. H. Spence, Advanced Transmission Electron Microscopy (Springer, New York, 2017).

[53] A. Genoni et al., Chem. Eur. J. 24, 10881 (2018).

[54] N. Ricardi, M. Ernst, P. Macchi, and T. A. Wesolowski, Acta Crystallogr. Sect. A 76, 571 (2020).

[55] D. Jayatilaka, Phys. Rev. Lett. 80, 798 (1998).

[56] D. Jayatilaka and D. J. Grimwood, Acta Crystallogr. Sect. A 57, 76 (2001).

[57] D. J. Grimwood and D. Jayatilaka, Acta Crystallogr. Sect. A 57, 87 (2001).

[58] L. Chęcińska, W. Morgenroth, C. Paulmann, D. Jayatilaka, and B. Dittrich, Cryst. Eng. Commun. 15, 2084 (2013).

[59] A. Genoni, L. H. R. Dos Santos, B. Meyer, and P. Macchi, IUCrJ 4, 136 (2017).

[60] M. Woińska, D. Jayatilaka, B. Dittrich, R. Flaig, P. Luger, K. Woźniak, P. M. Dominiak, and S. Grabowsky, ChemPhysChem 18, 3334 (2017).

[61] P. Coppens, X-ray Charge Densities and Chemical Bonding (Oxford University Press, New York, 1997).

[62] See Supplemental Materials at http://link.aps.org/ supplemental/10.1103/PhysRevLett.126.176402 for the full architecture of QCBED-DFT, all experimental details, DFT and QCBED settings within QCBED-DFT, refinement outputs, and discussions of materials properties and electronic structure morphologies. Additional references are cited [63-81].

[63] D. Peng, Ph.D. thesis, Monash University, 2020.

[64] D. M. Bird and Q. A. King, Acta Crystallogr. Sect. A 46, 202 (1990).

[65] H. P. Rooksby, Acta Crystallogr. 1, 226 (1948).

[66] Y. Shimomura, I. Tsubokawa, and M. Kojima, J. Phys. Soc. Jpn. 9, 521 (1954).

[67] C.W.F. T. Pistorius, Neues Jahrbuch für Mineralogie Monatshefte 11, 283 (1963).

[68] L. C. Bartel and B. Morosin, Phys. Rev. B 3, 1039 (1971).

[69] K. Momma and F. Izumi, J. Appl. Crystallogr. 44, 1272 (2011).

[70] A. Kokalj, J. Mol. Graphics Modelling 17, 176 (1999).

[71] H. A. Alperin, J. Phys. Soc. Jpn. 17, 1598 (1962).

[72] A. K. Cheetham and D. A. O. Hope, Phys. Rev. B 27, 6964 (1983).

[73] S. Kück and H. Werheit, in Landolt-Börnstein Group III Condensed Matter, edited by O. Madelung, U. Rössler, and M. Schulz (Springer, Berlin, 2000), Vol. 41D.

[74] S. Hüfner, J. Osterwalder, T. Riesterer, and F. Hulliger, Solid State Commun. 52, 793 (1984).

[75] G. A. Sawatzky and J. W. Allen, Phys. Rev. Lett. 53, 2339 (1984).

[76] Y. S. Grushko, Y. B. Paderno, K. Y. Mishin, L. I. Molkanov, G. A. Shadrina, E. S. Konovalova, and E. M. Dudnik, Phys. Status Solidi B 128, 591 (1985).

[77] F. Laves, Z. Phys. Chem., Abt. 22B, 114 (1933). 
[78] H. C. Longuet-Higgins and M. de V. Roberts, Proc. R. Soc. A 224, 336 (1954).

[79] S. Nakamura, T. Goto, S. Kunii, K. Iwashita, and A. Tamaki, J. Phys. Soc. Jpn. 63, 623 (1994).

[80] J. Duan, T. Zhou, L. Zhang, J. G. Du, G. Jiang, and H. B. Wang, Chin. Phys. B 24, 096201 (2015).

[81] S. Otani, H. Hiraoka, M. Ide, and Y. Ishizawa, JALCOM 189, L1 (1992).

[82] P. N. H. Nakashima and A. W. S. Johnson, Ultramicroscopy 94, 135 (2003).

[83] N. F. Mott, Proc. R. Soc. A 127, 658 (1930).

[84] H. A. Bethe, Ann. Phys. (N.Y.) 5, 325 (1930).

[85] H. A. Bethe, Ann. Phys. (Berlin) 392, 55 (1928).

[86] P. Blaha, K. Schwarz, F. Tran, R. Laskowski, G. K. H. Madsen, and L. D. Marks, J. Chem. Phys. 152, 074101 (2020).

[87] R. S. Pennington, C. Coll, S. Estradé, F. Peiró, and C. T. Koch, Phys. Rev. B 97, 024112 (2018).

[88] R. Adler, C. J. Kang, C. H. Yee, and G. Kotliar, Rep. Prog. Phys. 82, 012504 (2019).

[89] K. Lejaeghere et al., Science 351, aad3000 (2016).

[90] F. Tran, J. Stelzl, and P. Blaha, J. Chem. Phys. 144, 204120 (2016).

[91] V. I. Anisimov, J. Zaanen, and O. K. Andersen, Phys. Rev. B 44, 943 (1991).

[92] J. P. Perdew, K. Burke, and M. Ernzerhof, Phys. Rev. Lett. 77, 3865 (1996).

[93] S. Sato, J. Magn. Magn. Mater. 52, 310 (1985).

[94] A. A. Eliseev, V. A. Efremov, G. M. Kuzmicheva, E. S. Konovalova, V. I. Lazorenko, Y. B. Paderno, and S. Y. Khlyustova, Kristallografiya 31, 803 (1986).
[95] M. K. Blomberg, M. J. Merisalo, M. M. Korsukova, and V. N. Gurin, J. Less-Common Met. 168, 313 (1991).

[96] V. A. Streltsov, E. S. Konovalova, and Y. B. Paderno, Physica (Amsterdam) 259-261B, 1155 (1999).

[97] K. Tanaka and Y. Onuki, Acta Crystallogr. Sect. B 58, 423 (2002).

[98] S. L. Dudarev, G. A. Botton, S. Y. Savrasov, C. J. Humphreys, and A. P. Sutton, Phys. Rev. B 57, 1505 (1998).

[99] V. I. Anisimov, I. V. Solovyev, M. A. Korotin, M. T. Czyżyk, and G. A. Sawatzky, Phys. Rev. B 48, 16929 (1993).

[100] P. Wei and Z. Q. Qi, Phys. Rev. B 49, 10864 (1994).

[101] J. Hugel and M. Kamal, J. Phys. Condens. Matter 9, 647 (1997).

[102] S. K. Kwon and B. I. Min, Phys. Rev. B 62, 73 (2000).

[103] G. K. H. Madsen and P. Novák, Europhys. Lett. 69, 777 (2005).

[104] M. Cococcioni and S. de Gironcoli, Phys. Rev. B 71, 035105 (2005).

[105] T. Cai, H. Han, Y. Yu, T. Gao, J. Du, and L. Hao, Physica (Amsterdam) 404B, 89 (2009).

[106] C. K. Barman, P. Singh, D. D. Johnson, and A. Alam, Phys. Rev. Lett. 122, 076401 (2019).

[107] P. A. Doyle and P. S. Turner, Acta Crystallogr. Sect. A 24, 390 (1968).

[108] D. T. Cromer and J. T. Waber, in International Tables for X-Ray Crystallography, Vol. 4, edited by J. A. Ibers and W. C. Hamilton (Kynoch, Birmingham, 1974), pp. 71-147.

[109] P. J. Brown, A. G. Fox, E. N. Maslen, M. A. OKeefe, and B. T. M. Willis, in International Tables for Crystallography, 3rd ed., edited by E. Prince (IUCr and Wiley, Hoboken, NJ, 2006), Vol. C, Chap. 6.1, pp. 554-595. 